\title{
On the refractoriness of somesthetic temporal acuity'
}

\author{
WILLIAM R. UTTAL AND MADELON KRISSOFF \\ THE UNIVERSITY OF MICHIGAN
}

Preconditioning with a burst of electrical pulse stimuli elevates the threshold for temporal acuity as measured with a technique requiring the $S$ to detect a short gap in an otherwise regular burst of similar stimuli. Only a small amount of backward inhibition can be observed. The temporal duration of the inhibitory effect (up to $700 \mathrm{msec}$.) suggests that repetitive after-discharges of second-order afferents in the spinal cord may underlie this behavioral measure by actually filling in the intervals which are used by the $S$ to make the judgment. The phenomenon is thus thought to be a disruption of a true temporal judgment rather than one indirectly mediated by an amplitude judgment, and as such it represents a signific ant datum on the temporal resolving power of time dimensions of neural coding.

One of the problems of continuing interest to sensory psychologists has been the effect of multiple stimuli on each other. By defining the interactions of simple stimuli which differ only in time or place of occurrence, it has often proven possible to provide explanations for what had hitherto been considered irreducible "subjective" phenomena. An excellent example of this is the definitive physiological explanation of the Mach band on the basis of spatial interaction in, of all things, the eye of the horseshoe crab. (See Ratliff, 1965.)

In this paper we are concerned with the temporal interaction of sequential bursts of impulsive stimuli. One may distinguish several different categories of research exploring the temporal interactions of serial stimuli. The first category deals with those serial interactions in which the subjective magnitude of a sensation or the amplitude threshold is altered by either by a preceding or a succeeding stimulus. In somatosensation this sort of experiment is best illustrated by the studies of inhibitory interaction which Sherrick (1966) has pointed out to be analogous to auditory studies of masking. The studies of Schmid (1961), Rosner (1964), Sherrick (1964), and Uttal (1960) on the somatosensory system all explore the effect of masking stimuli on the absolute magnitude threshold of a test stimulus. In the case of Rosner's experiment in which the conditions for inhibitory interaction were optimized-both test and masking stimuli were applied to the same location-this inhibitory interaction was found to last as long as $250 \mathrm{msec}$.

The question to which we presently turn concerns another category of serial interaction. We report the results of some experiments on the recovery cycle of inhibitory effects on temporal acuity as measured by the S's ability to detect a gap in a repetitive train of stimuli.

It has already been shown that temporal acuity is extremely susceptible to the temporal pattern within the stimulus train (Uttal \& Krissoff, 1966), or even to the relative amplitude of each of two pulses (Gescheider, 1966). The current experiment describes the elongated recovery cycle of temporal acuity following a conditioning burst of stimuli and the effect of the number of pulses in the conditioning burst on this recovery cycle.

We also present the results of a test of an equivalent backward masking paradigm. A possible physiological mechanism for the forward masking condition is discussed.

\section{Subjects}

\section{METHOD}

The data presented in Fig. 2 are composed of the pooled responses of five Ss; in Fig. 3, of five Ss; and in Fig. 6, of three Ss. It should be noted that considerable intersubject variability exists, but the general form of these results has been replicated with other groups of Ss.

\section{Apparatus}

The stimulus trains presented in these and other experiments consisted of groups of constant current electrical pulses $.5 \mathrm{msec}$. in duration with an amplitude of $4 \mathrm{~mA}$. The trains of stimulus pulses were fed, via silver electrodes, to a pair of test tubes filled with body normal saline solution into which the $S$ inserted the first joint of the index and middle fingers of his left hand. The timing of the stimulus train was controlled by a small digital computer which also acquired the responses made by the $\mathrm{S}$. These data were analyzed with simple statistical measures which were displayed immediately following the protocol of the S's responses. The S, seated in a soundproof enclosure, was instructed to report whether there was a discontinuity or "gap" in the test portion of the stimulus train. The stimuli were reported by the $S$ to be intermittent buzzes and produced no observable motor response.

As described in our earlier paper (Uttal \& Krissoff, 1966), the psychophysical method used was a computercontrolled up and down method of limits. If the $S$ reported the gap to be present, the computer program was designed to shorten the gap for the next presentation. If he reported it to be absent, the gap was automatically elongated. 


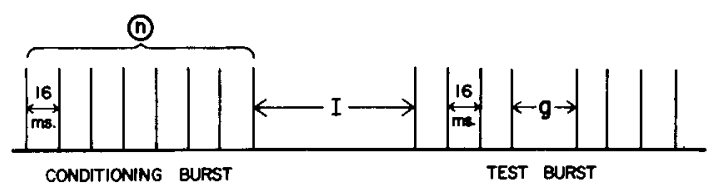

Fig. 1. The organization of a typical stimulus pattem showing the number of pulses in the conditioning burst ( $n$ ), the interval between the conditioning and test burst (I), and the gap within the test burst ( $\mathrm{g}$ ).

\section{Procedure}

The main purpose of this experiment was to explore the recovery cycle of temporal acuity as a function of the interval between the conditioning burst and the test burst. A secondary goal was to determine the differential inhibitory effect of the number of pulses in the conditioning burst. A typical stimulus patternone presentation-was organized as shown in Fig. 1. In this figure can be seen the standard test burst of eight pulses and one possible conditioning burst, which in this cáse is also composed of eight pulses. The pulses comprising the standard test burst and the conditioning burst were always separated from each other by $16 \mathrm{msec}$. except for the gap. The gap size (g), continuously tracked by the computer program, represented the dependent variable measured in this experiment.

Conditioning bursts composed of one, four, and eight pulses tested the effects of numerosity on temporal acuity. (An additional set of data was run for the condition in which the conditioning burst contained six pulses. This was run on a smaller $\mathrm{S}$ sample containing only three Ss, and the data are therefore presented only as a supplement to our discussion.)

Trials were presented in blocks of 75 . Within each block, it should be remembered, the sequence of the gap sizes was dependent upon the S's pattern of responses and is therefore not explicitly describable. The initial gap presented for the first block each day was 100 msec.-well above any of the measured thresholds. The first few responses were used by the $\mathrm{S}$ to locate the threshold region. Therefore, the first nine trials in a block were always excluded from the data analysis to eliminate any effects of this initial search transient. The initial gap for subsequent blocks was set equal to the average gap size of the preceding block. The interval between the conditioning and test bursts was held constant within each block, but the seven intervals were presented in a constrained random order of seven blocks each day for seven days. The three conditions of numerosity were run in different orders for the five Ss. With this experimental design it can be seen that each data point plotted in Fig. 2 is the average of 2,310 judgments by the Ss.

As a supplementary experiment we tested for the presence of backward conditioning by repeating the experiment with five Ss. In this experiment the con- ditioning burst followed the test burst and was composed of either one or eight pulses at an interval of $100,200,300$, or $400 \mathrm{msec}$. following the test burst. All other conditions of the experiment remained the same except that the Ss were instructed to detect a gap in the first rather than in the second burst.

\section{RESULTS}

The results of this experiment are presented in Fig. 2. The horizontal coordinate represents the intervals between the conditioning and the test bursts. The vertical coordinate represents the mean threshold in milliseconds for the detection of the gap in the test burst, and the three curves represent the three different numbers of pulses in the conditioning bursts. The first result to be noted is that there is a substantial inhibition of the ability to make the temporal discrimination and that this inhibitory interaction decreases with increases in the interval between the conditioning and the test bursts. The inhibitory effect appears to last for as long as $600 \mathrm{msec}$. Compared to the amplitude-masking studies mentioned in the introduction, this is an extraordinarily long time.

Perhaps even more striking, though, is the unexpected result of the elevation of the threshold when the conditioning burst consisted of only a single pulse. It appears that the inhibitory effect for this condition of $\mathrm{n}$ is much greater than in either of the other two conditions. We believe this elevation to be an artifact, however, and we will discuss this point in the following section.

Another notable feature of the data is the cross-over of the two curves representing the effects of the other two parameters of conditioning burst numerosity. We also believe this result to be an artifact in the sense to be discussed below.

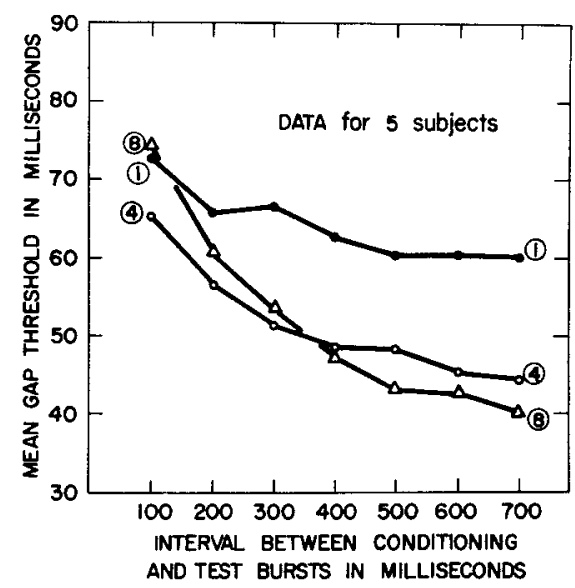

Fig. 2. Data for the forward conditioning situation for 5 subjects showing the results of the experiment before normalization. Mean gap threshold is plotted as a function of $I$. The 3 curves are parametric in $n$, the number of stimulus pulses in the conditioning burst. 


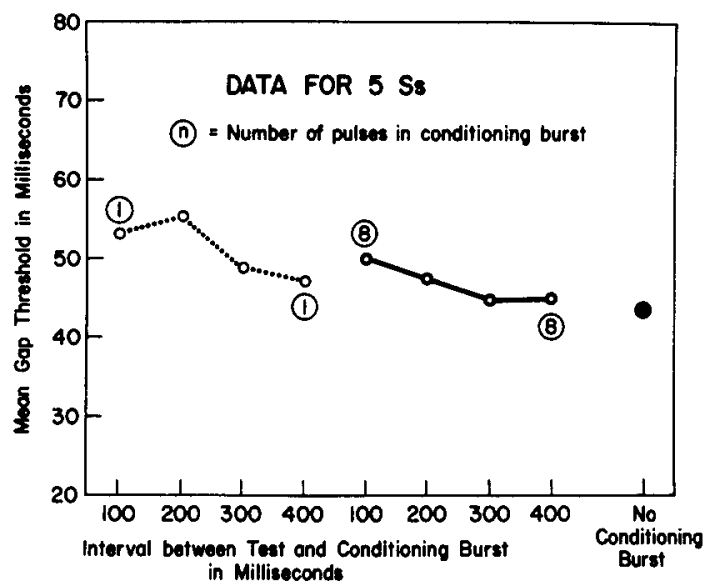

Fig. 3. Data for the backward conditioning situation. The 2 curves are parametric in $n$, the number of stimulus pulses in the following conditioning burst.

Figure 3 presents the results of the experiments designed to test for backward conditioning. A slight inhibitory effect appears for the shorter intervals, although it is seen to be far less strong than the one produced by a preceding conditioning burst. There is also, in this case, a slight suggestion that the threshold was elevated more by the single pulse than by the eight-pulse conditioning burst.

\section{DISCUSSION}

The results of this experiment clearly show that a preceding burst substantially inhibits the discriminatory capacity called temporal acuity. The effect generally tends to increase as the interval between a conditioning and a test burst decreases. The length of time over which these effects are exhibited is considerably longer than the only superficially comparable magnitude masking effects described above.

The main issues to be discussed regarding these results concern the apparent super-effectiveness of a single pulse as an inhibitor and the cross-over of the other two curves. To understand these, it is necessary to consider the results of one of the experiments discussed in our earlier paper (Uttal \& Krissoff, 1966). The experiment (No. III) dealt with the effect of the number of pulses in the stimulus train on the gapdetection threshold with no conditioning burst. The results of that experiment are replotted in Fig. 4. Note that the size of the gap between four, six, eight, ten, and twelve pulses was relatively constant. Nevertheless, our Ss did have a greatly elevated threshold for the situation in which they were asked to detect a gap between only two pulses. However, Ss naive to the long pulse bursts of our experiments display a much lower two-pulse threshold than those who have experienced these long stimuli. Thus it seems likely that the elevation of this one point on Fig. 4 is, in a sense, an artifact of our particular experimental paradigm.

It is possible to suggest several alternative reasons

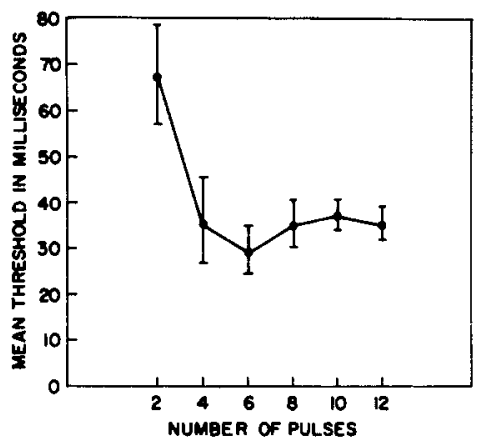

Fig. 4. Data from the earlier study (Uttal \& Krissolf, 1966), showing the elevation of the gap threshold when there were only 2 pulses in the test burst and no conditioning burst. See text for more complete explanation of these data.

why this might occur, but such speculation serves little purpose in the context of this discussion. It is more direct to state operationally that the S probably changed the criterion on which his decision was based in the different instances in which he was called upon to resolve two pulses. We believe that the apparent super-inhibitory effect of the single pulse in the present experiment also represents just such a criterion shift on the part of the S. Therefore, the absolute levels of the inhibition are not truly comparable. In the same way, the cross-over of the other two curves probably also represents a shift of criterion level and our results can not, therefore, be compared on an absolute level basis.

We have performed a simple normalization of the data of Fig. 2 to allow a more direct comparison of the recovery cycle effects in which we are most interested. All data points have been divided by the threshold size of the gap for an interval of $700 \mathrm{msec}$. This allows us to replot our data as shown in Fig. 5. In this figure the vertical coordinate now represents

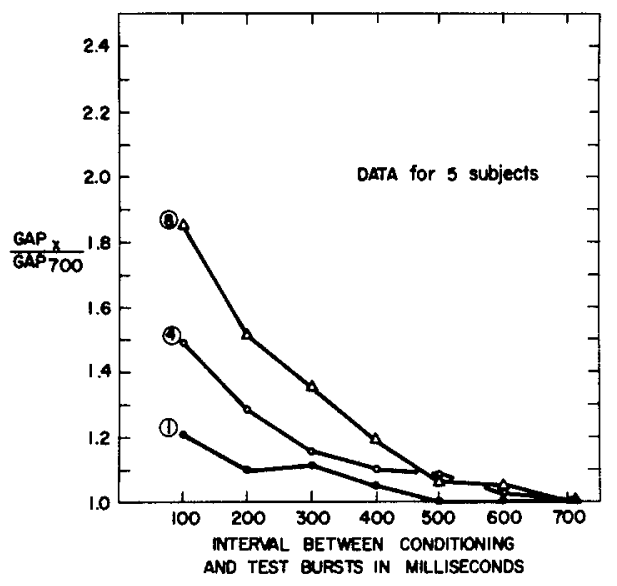

Fig. 5. Normalized data for 5 subjects for the forward conditioning situation. 


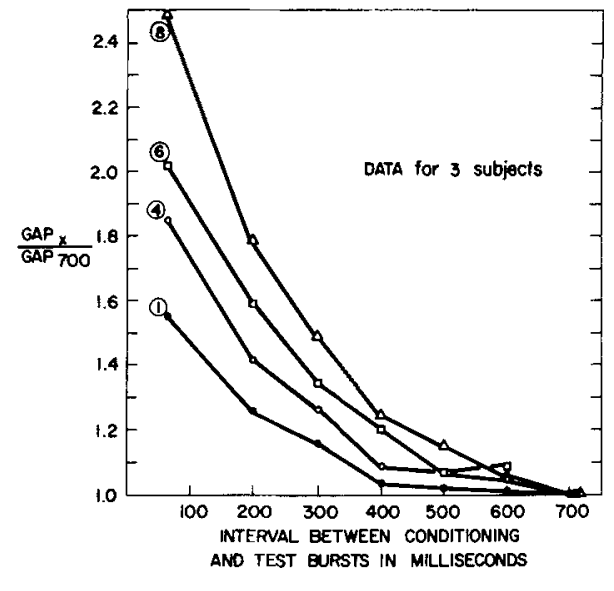

Fig. 6. Data for a smaller sample of subjects (3), showing the normalized form of the data for 4 values of $n$.

the threshold for the test gap expressed as a dimensionless ratio with respect to this intrinsic standard of the mean gap size for an I of $700 \mathrm{msec}$. In this context the results can now be seen to be more simply organized with regularly increasing inhibitory effects as a function of both the number of pulses in the conditioning burst and the decreasing interval between conditioning and test bursts.

To support further the notion that these data do not represent spurious functions due to our sampling, we have also plotted the results of a replication of our basic experimental paradigm for a smaller (three) sample of Ss in Fig. 6. We have included additional data for the situation in which the conditioning burst consisted of six pulses. The curve for this new value of $n$ falls, as expected, between the curves for n's of 4 and 8 .

Is the notion valid that the inhibitory effect exhibited is a true disruption of an ability to deal with time as such, rather than an effect of a reduction in the subjective amplitude of one of the pulse bursts? A considerable amount of evidence supports this former notion. Our stimulus amplitudes were about 3-4 times the amplitude of the absolute threshold reported elsewhere for this kind of electrical stirnulus. Furthermore, our Ss never spontaneously reported any difficulty in detecting any of the parts of the two-stimulus bursts, even when probed with direct questions. Another fact supporting the contention that a disruption of temporal acuity occurred, resulted from our earlier study (Uttal \& Krissoff, 1966). There it was shown that for the situation in which there was no conditioning shock, the stimulus amplitude-and thus the subjective amplitude-did not affect the threshold size of the gap within the range of $2-6 \mathrm{~mA}$.

In the light of this evidence, we conclude that our effect is a disruption of a true temporal judgment with an unusually elongated recovery cycle. This statement is not definitive in a physiological sense, for a disruption of the time sense could occur because of a peripherally noisy information channel or because the central decoding mechanism is itself disrupted.

The relatively small amount of backward inhibition suggests that this effect is one which can be best described in terms of the brief neural transients of the peripheral nervous system. Frank and Fuortes (1956) have described a peripheral neural phenomenon which could account for our results. They studied the after-discharge of spinal interneurons in the cat and found that spontaneously active fibers or fibers which responded with repetitive bursts to a single afferent volley would produce a greater degree of afterdischarge following a repetitive stimulus train. From their records it appears that this activity continues for about a second following the stimulus train. Such after-discharges in the human second-order somatosensory neurons could serve to fill in the intervals between stimuli on which the acuity judgment made by our Ss is based, and thus they could provide a simple and relatively peripheral explanation of the elevation of the threshold described in this paper.

\section{References}

Frank, K., \& Fuortes, M. G. F. Unitary activity of spinal interneurones of cats. J. Physiol., 1956, 131, 424-435.

Gescheider, G. A. Resolving of successive clicks by the ears and skin. J. exp. Psychol., 1966, 71, 378-381.

Ratliff, F. Mach bands: quantitative studies on neural netuorks in the retina. San Francisco: Holden-Day, Inc., 1965.

Rosner, B. S. Temporal interaction between electrocutaneous stimuli. J, exp. Psychol., 1964, 67, 191-192.

Schmid, Ethel. Temporal aspects of cutaneous interaction with two-point electrical stimulation. J. exp. Psychol., 1961, 61, 400-409.

Sherrick, C. E. Effects of double simultaneous stimulation of the skin. Amer. J. Psychol., 1964, 77, 42-53.

Sherrick, C. E. Somesthetic senses. Annu. Rev. Psychol., 1966 , $17,309-336$.

Uttal, W. R. Inhibitory interaction of responses to electrical stimuli in the fingers. J. comp. physiol. Psychol., 1960, 53, 47-51.

Uttal, W. R., \& Krissoff, Madelon. Effect of stimulus pattern on temporal acuity in the somatosensory system. J. exp. Psychol., $1966,71,878-883$.

\section{Note}

1. The research reported in this paper was supported in part by NIH grant 08786-02 and in part by NSF grant GB-2000.

(Accepted for publication Decenber 27, 1966.) 